\title{
Mécanisme de l'action hypocorticostéronémiante de Strongyloides ratti chez le Rat
}

\author{
par J. BAILENGER et G. FARAGGI \\ Laboratoire de Zoologie et Biologie animale, Faculté de Pharmacie, \\ place de la Victoire, F 33000 Bordeaux
}

\section{Résumé.}

Afin d'expliquer l'hypocorticostéronémie qui s'établit chez les rats parasités par Strongyloides ratti, nous effectuons une exploration fonctionnelle de l'axe hypothalamo-hypophyso-corticosurrenal chez des rongeurs expérimentalement infestés.

L'épreuve de l'A.C.T.H., le stress à l'éther ainsi que les tests à la Lysine-8-Vasopressine et à la Métopirone fournissent des résultats concordants :

- le complexe hypothalamus-pituitaire-corticosurrénal présente une intégrité fonctionnelle qui lui permet de réagir normalement à une stimulation directe ou indirecte ; physotrope ;

- la déficience semble porter sur la sécrétion hypothalamique du facteur hypo-

- la cause résiderait dans une inhibition du mécanisme nerveux qui contrôle cette neurosécrétion.

\section{Summary.}

Mechanism of the hypocorticosteronemic action of Strongyloides ratti in the Rat.

In order to explain the hypocorticosteronemia which establishes itself in rats with Strongyloides ratti parasites, we are proceeding with a functional exploration of the hypothalamo-hypophyso-corticosurrenal axis in experimentally infested rodents.

The adreno-cortico-tropic-hormone test (A.C.T.H.), stress to ether and the Lysin-8Vasopressin and Metopirone tests lead to concordant results :

- the hypothalamo-pituitary-corticosurrenal present a functional integrity which allows it to react normally to direct stimulation or to feed-back; 

factor ;

- the deficiency seems to relate to the hypothalamic secretion of the cortico-releasing

- the cause would stem from an inhibition of the nervous mechanism which regulates this neurosecretion.

Bailenger et Carcenac (1973) ont montré que Strongylö̈des ratti se comporte, dans l'organisme des rats, comme l'agent d'une agression non spécifique déterminant, après une élévation fugace et précoce de la corticostéronémie, une baisse prolongée du taux plasmatique de cette hormone.

Pour aborder le mécanisme de cette action hypocorticostéronémiante, nous nous proposons d'explorer les différents constituants de l'axe hypothalamo-hypophyso-corticosurrénal des rats infestés, afin d'essayer de localiser la défaillance fonctionnelle provoquée par le parasite.

Nous dissocions :

- Le cortex surrénal : test de l'A.C.T.H.

- Le complexe hypothalamo-pituitaire : test à l'éther ; test à la Lysine-8-Vasopressine ; test à la Métopirone.

\section{Matériel et méthodes}

Dans tous les cas, ces épreuves sont accomplies sur des rats infestés expérimentalement par Strongyloïdes ratti et à la période où leur hypocorticostéronémie est la plus marquée.

Les rats utilisés dans les différentes expériences appartiennent à la souche Wistar ; ils sont pathogen-free et de sexe mâle. Lorsqu'ils atteignent $250 \mathrm{~g}$, on les infeste par balnéation en les maintenant pendant 50 minutes dans un bocal de 2 litres renfermant $60 \mathrm{ml}$ d'eau tenant en suspension des larves infestantes en nombre exactement déterminé, mais variable selon les expériences et suffisant pour réaliser une contamination toujours intense.

Tous les rats sont maintenus isolés et placés dans des conditions rigoureusement identiques pour éviter l'interférence des agressions dues à l'environnement.

Le contrôle du parasitisme et le dosage de la corticostérone ont été décrits (Bailenger et Carcenac, 1973), ainsi que les prélèvements sanguins qui sont faits régulièrement à 9 heures pour tenir compte du cycle nycthéméral qui caractérise la physiologie de l'axe hypothalamo-hypophyso-surrénal.

Les animaux sont répartis en trois ou quatre lots: l'un infesté que l'on réserve pour le soumettre au test au moment opportun; le deuxième lot, également infesté, que l'on subdivise en plusieurs groupes pour suivre les variations de la corticostéronémie en se mettant à l'abri du stress provoqué par des prélèvements sanguins répétés. Les témoins comportent 1 ou 2 lots selon les expériences. 
Epreuves à l'A.C.T.H. : nous avons choisi un Tetracosactide: polypeptide de synthèse contenant les 24 premiers acides aminés de l'A.C.T.H. et réputé pour ne pas provoquer de réactions allergiques. Adsorbé sur de l'hydroxyde de zinc colloïdal, le produit est ainsi administré sous une forme retard, par voie sous-cutanée, à raison de deux injections de $0,3 \mathrm{mg}$ de Tetracosactide-zinc par kilo de poids. Les deux injections sont espacées de 24 heures.

Test à l'éther : l'éther est bien connu comme un stimulus puissant pour la libération de l'A.C.T.H., provoquant une élévation précoce de la corticostérone dans le sang. L'augmentation sanguine de l'A.C.T.H. se manifeste 2 minutes à 2 minutes 30 après inhalation d'éther. Une étude précise des répercussions du stress à l'éther sur le taux de la corticostéronémie chez le Rat a été faite par Beigelman (1956), Kitay (1961) et Hedner (1961-1962).

En pratique, le test à l'éther comporte un séjour de 3 minutes des animaux dans une atmosphère d'éther suivi, 30 minutes plus tard, d'un prélèvement de sang dans lequel la corticostérone est dosée.

Le test à la Lysine-8-Vasopressine a été proposé par Gwinup (1965) ainsi que par Van der Wal (1965) pour explorer l'activité exercée par l'hypothalamus sur la sécrétion anté-hypophysaire.

En l'absence de documents sur la réalisation de cette épreuve chez le Rat, des essais préliminaires ont été nécessaires pour en préciser les conditions optima.

La Lysine-8-Vasopressine est injectée par voie intra-musculaire sous forme d'un soluté à 1 unité/ml préparé par dilution du produit commercial dans une solution d'acide chlorhydrique $0,01 \mathrm{~N}$ à 9 p. 1.000 de chlorure de sodium. En tenant compte des travaux de Wied (1964) et de Cheiffetz (1969), nous avons adopté la dose de 100 milli-unités par $100 \mathrm{~g}$ de poids corporel. En l'absence de renseignements sur le temps de réponse de la corticosurrénale des rats, nous l'avons défini expérimentalement en effectuant les prélèvements de sang, à des lots différents de rats, 15, 30 et 60 minutes après injection de Lysine-Vasopressine. La sécrétion est maximale au bout de 30 minutes. Clayton (1963) aboutit à une conclusion similaire chez l'homme.

Le moment de la réalisation de ce test ne semblant pas intervenir, d'après Clayton, nous l'avons réalisé à $9 \mathrm{~h}$ du matin.

Test à la Métopirone : cette molécule, encore dénommée Métyrapone, Méthyrapone ou SU 4885, inhibe la 11- $\beta$-hydroxylation qui conduit à la corticostérone. La synthèse de cette hormone est donc bloquée au stade de 11-désoxycorticostérone ou Cortexone qui n'exerce pas de rétrocontrôle sur la sécrétion d'A.C.T.H.

Normalement, l'administration de Métopirone entraîne donc une hypocorticostéronémie qui libère la sécrétion d'A.C.T.H. d'où une élévation du taux de Cortexone.

Le test à la Métopirone fournit donc des indications sur l'intégrité du contrôle * feed-back » vis-à-vis de la sécrétion des corticostimulines.

La réalisation du test est bien mise au point chez l'homme, mais les éléments dont on dispose pour le Rat manquent de précisions et ne concernent pas l'exécution 
d'un test d'exploration. Les problèmes que nous avons dû résoudre pour l'appliquer à cet animal ont été multiples.

En tenant compte des données bibliographiques et des résultats apportés par des expériences préliminaires, nous avons adopté les conditions suivantes:

$1^{\circ}$ La Métopirone est mise en solution à 10 p. 100 dans une solution aqueuse stérile à 10 p. 100 d'acide ascorbique.

La solution est injectée par voie intra-péritonéale; la première expérience comporte 2 injections, à 2 heures d'intervalle, chacune d'elles correspondant à $100 \mathrm{mg}$ de Métopirone par kilo de poids corporel. Dans la deuxième expérience, on fait 4 injections de $125 \mathrm{mg} / \mathrm{kg}$, espacées toutes les 3 heures.

$2^{\circ}$ Le sang est prélevé 2 heures après la dernière injection. Il permet, d'une part, un dosage fluorescimétrique de la Corticostérone dont le taux doit être considérablement abaissé si le blocage a été réalisé et, d'autre part, on y recherche quantitativement la $11-\beta$-désoxycorticostérone dont l'apparition permet de suivre la réponse hypothalamo-pituitaire, c'est-à-dire d'apprécier les possibilités de ce complexe de réagir à une chute de la corticostérone circulante.

$3^{\circ}$ Recherche et dosage de la 11- $\beta$-désoxycorticostérone : la fluorescimétrie en milieu sulfurique ne permet pas de doser le cortexone puisque les corticostéroïdes ne présentant pas d'OH en 11 ne sont que très faiblement fluorescents. De plus, il ne s'agit pas d'un 17-OH-stéroïde, de sorte que l'on ne peut appliquer les méthodes utilisées en médecine humaine. Nous nous sommes donc orientés vers la chromatographie ascendante en couche mince sur silicagel.

Les gluco-cortico-stérö̈des sont extraits du plasma $(3 \mathrm{ml})$ en l'agitant, pendant une minute, avec $15 \mathrm{ml}$ de Dichlorométhane. Après lavage rapide (agitation de 10 secondes) du solvant avec $1 \mathrm{ml}$ de soude $0,1 \mathrm{~N}$, on prélève $12 \mathrm{ml}$ de la phase organique que l'on évapore sous courant d'azote. Le résidu est dissous dans deux gouttes d'un mélange à volumes égaux chloroforme-éthanol absolu.

Cette solution est soumise à une chromatographie ascendante sur gel de Silice en utilisant comme solvant un mélange acétate d'éthyle-cyclohexane (8/2). Après séchage, les taches sont révélées par le bleu de Tétrazolium.

Des dépôts témoins de $0,25 \mu \mathrm{g}, 0,5 \mu \mathrm{g}$ et $1 \mu \mathrm{g}$ de Corticostérone et de désoxycorticostérone forment des taches de référence.

$4^{\circ}$ La réalisation du test à la Métopirone doit tenir compte du rythme circadien de la sécrétion d'A.C.T.H. qui, chez les rongeurs, est l'inverse de celui de l'homme et tel que le taux de la corticostérone est maximum l'après-midi.

En effectuant ce test à 3 périodes différentes de la journée, tel que les prélèvements de sang soient effectués à 10 heures, 14 heures et 19 heures, nous avons pu nous rendre compte que la réponse la plus forte était enregistrée à 14 heures et la plus faible à 10 heures. Cette observation est en accord avec les données physiologiques concernant la périodicité de la réponse hypophyso-pituitaire qui, pour les rats, présente un maximum l'après-midi. 
$5^{\circ}$ Les animaux sont répartis en 4 lots. Deux d'entre eux sont infestés. Leur parasitisme est suivi par des analyses coprologiques. Sur l'un, on établit l'évolution de la corticostéronémie; le test à la Métopirone est effectué sur l'autre au moment où l'hypocorticostéronémie est la plus marquée. Les deux autres lots ne sont pas infestés et servent de témoins.

\section{Résultats}

Epreuve à l'A.C.T.H. (tableau I).

La mise en évidence d'un état d'hypofonctionnement éventuel du cortex surrénal est réalisée en sollicitant expérimentalement cette glande par l'Adreno-corticotropichormon (A.C.T.H.).

TABleau I. - Test à l'A.C.T.H.

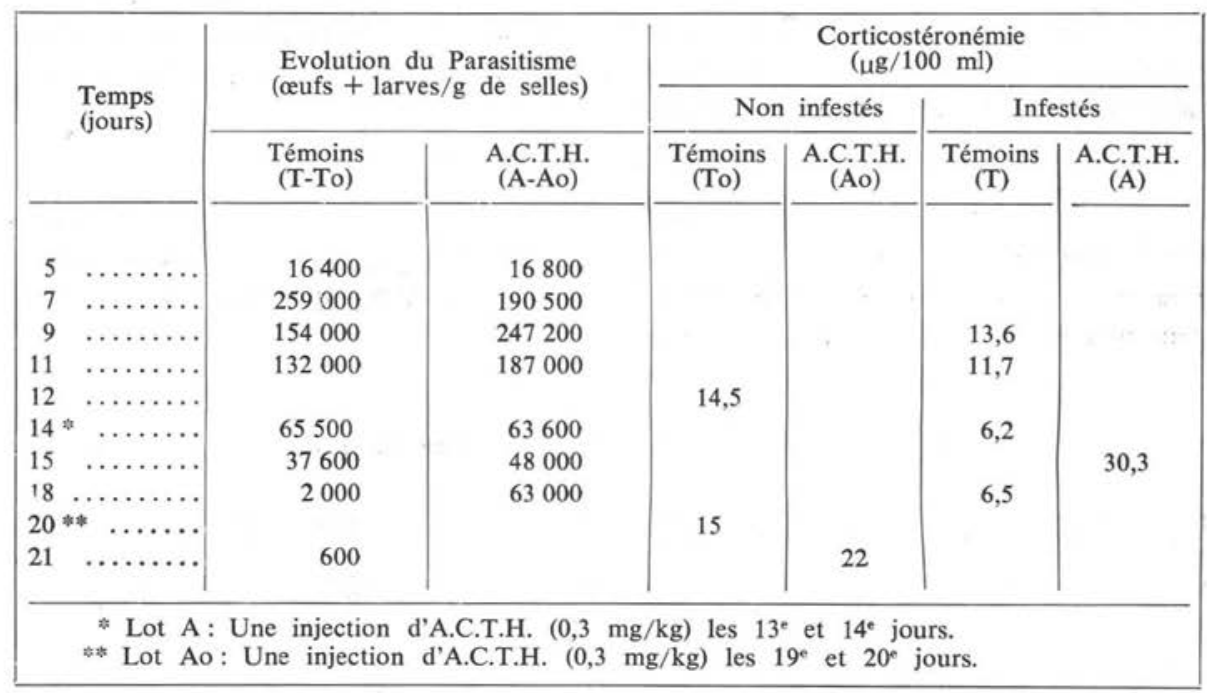

24 rats sont répartis en trois lots :

Huit animaux sont parasités par 12000 larves ; leur corticostéronémie s'abaisse à $6,2 \mu \mathrm{g}$ au $14^{\mathrm{e}}$ jour. C'est à ce moment qu'est effectué le test à l'A.C.T.H. sur 6 rats également infestés par 12000 larves; seize heures après la seconde injection de Tetracosactide-zinc, la corticostérone passe de $6 \mu \mathrm{g}$ à $30,3 \mu \mathrm{g}$.

Dix rats ne sont pas infestés et servent de témoins; leur corticostéronémie évolue autour de $15 \mu \mathrm{g}$. La réalisation du test à l'A.C.T.H., dans les mêmes conditions que celles retenues pour les animaux infestés, fait monter leur corticostéronémie à $22 \mu \mathrm{g}$. 


\section{Test à l'éther.}

Six rats non infestés permettent de suivre le taux normal de la corticostérone dans l'expérience en cours; au quinzième jour de l'expérience, la corticostérone d'un lot de 8 rats infestés par 12000 larves atteint un niveau très bas à $4 \mu \mathrm{g} / 100 \mathrm{ml}$ de plasma. Le test à l'éther est effectué à cette période parallèlement sur les animaux parasités et les témoins mis en réserve et respectivement aux nombres de 8 et 6 : la corticostéronémie s'élève à 40,1 $\mu \mathrm{g}$ (parasités) et 29,1 $\mu \mathrm{g}$ (témoins).

Le parasitisme des rats soumis à cette épreuve avait atteint un maximum au $8^{e}$ jour avec 326000 œufs et larves dans les selles. La self-cure s'est produite aux environs du $20^{\circ}$ jour.

\section{Test à la Lysine-8-Vasopressine.}

Trois séries expérimentales ont été réalisées. Pour les deux premières, la dose de L.V.P. injectée est proportionnelle au poids des rats. Pour la troisième, une variante posologique est introduite: la quantité de L.V.P. est la même pour tous les animaux indépendamment de leur poids individuel.

Toutes les infestations sont faites par 15000 larves.

Expérience $\mathrm{n}^{\circ} 1$ (tableau II). 60 animaux (40 infestés ; 20 non infestés) : l'épreuve à la Lysine-Vasopressine a lieu au $15^{\circ}$ jour du parasitisme, la corticostéronémie s'étant abaissée à 4,5 $\mu$ g. Trente minutes après l'injection de L.V.P., elle passe de 8 à $54 \mu \mathrm{g}$ (témoins) et de 4,5 à $37 \mu \mathrm{g}$ (parasités).

TABleau II. - Test à la Lysine-8-Vasopressine (Epreuve $\mathrm{n}^{\circ}$ 1)

\begin{tabular}{|c|c|c|c|c|c|}
\hline & \multirow{2}{*}{$\begin{array}{l}\text { Temps } \\
\text { (jours) }\end{array}$} & \multicolumn{2}{|c|}{$\begin{array}{l}\text { Evolution du Parasitisme } \\
\text { (œufs + larves/g. de selles) }\end{array}$} & \multicolumn{2}{|c|}{$\begin{array}{l}\text { Corticostéronémie } \\
(\mathrm{ug} / 100 \mathrm{ml})\end{array}$} \\
\hline & & Témoins & L.V.P. & Non infestés & Infestés \\
\hline 0 & $\ldots \ldots \ldots \ldots \ldots$ & & & 12 & 12 \\
\hline 6 & $\ldots \ldots \ldots \ldots \ldots$ & 126200 & 89200 & & \\
\hline 8 & $\ldots \ldots \ldots \ldots \ldots$ & 150000 & 134000 & & \\
\hline 10 & n.m. & 112000 & 94800 & 8,2 & 6,3 \\
\hline 13 & $\ldots \ldots \ldots \ldots \ldots$ & 104000 & 94600 & & \\
\hline 14 & $\ldots \ldots \ldots \ldots \ldots$ & & & 8 & 4,5 \\
\hline 15 & $\ldots \ldots \ldots \ldots \ldots$ & 29000 & 60000 & 54 * & $37^{*}$ \\
\hline 16 & $\ldots, \cdots, \cdots$ & & & & 4,6 \\
\hline 17 & $\ldots \ldots \ldots \ldots \ldots$ & 11000 & 6800 & & \\
\hline 20 & $\ldots \ldots \ldots \ldots$ & 300 & 0 & & \\
\hline
\end{tabular}


Expérience $\mathrm{n}^{\circ} 2$ (tableau III). 30 animaux (15 infestés et 15 non infestés): au $14^{\circ}$ jour de l'infestation, le plasma renferme $8 \mu \mathrm{g}$ de corticostérone ; celui des témoins en contient 17. Le test à la L.V.P. est effectué à cette période : la corticostéronémie s'élève à $74,3 \mu \mathrm{g}$ chez les rats non parasités et à $63 \mu \mathrm{g}$ chez les rats infestés.

TABLEAU III. - Test à la Lysine-8-Vasopressine (Epreuves $\mathrm{n}^{\circ \times} 2$ et 3 )

\begin{tabular}{|c|c|c|c|c|c|}
\hline & \multirow{2}{*}{$\begin{array}{l}\text { Temps } \\
\text { (jours) }\end{array}$} & \multicolumn{2}{|c|}{$\begin{array}{l}\text { Evolution du Parasitisme } \\
\text { (æufs + larves/g. de selles) }\end{array}$} & \multicolumn{2}{|c|}{$\begin{array}{l}\text { Corticostéronémie } \\
(\mu \mathrm{g} / 100 \mathrm{ml})\end{array}$} \\
\hline & & Témoins & L.V.P. & Non infestés & Infestés \\
\hline 0 & $\ldots \ldots \ldots \ldots \ldots$ & & & 19,7 (To Ao) & \\
\hline 5 & $\ldots \ldots \ldots \ldots$ & 19200 & 12000 & & 8,3 (Ao) \\
\hline 7 & $\ldots \ldots \ldots \ldots$ & 511000 & 355000 & 9,3 (To) & \\
\hline 9 & $\ldots \ldots \ldots \ldots$ & 254000 & 307000 & & \\
\hline 12 & $\ldots \ldots \ldots \ldots$ & 129000 & 126000 & & \\
\hline 14 & $\ldots \ldots \ldots \ldots \ldots$ & 72000 & 39600 & 17 (To) & 8 (Ao) \\
\hline 16 & $\ldots \ldots \ldots \ldots$ & 20800 & 29600 & $74,3 *\left(T_{1}\right)$ & $63 \div\left(A_{1}\right)$ \\
\hline 19 & $\ldots \ldots \ldots \ldots \ldots$ & 2000 & 3600 & & \\
\hline 20 & $\ldots \ldots \ldots \ldots$ & & & 77,8 해 $\left(T_{2}\right)$ & 58,8 के $\left(\mathrm{A}_{2}\right)$ \\
\hline 21 & $\ldots \ldots \ldots \ldots \ldots$ & 400 & 2000 & 12 (To) & 9,3 (Ao) \\
\hline 23 & $\ldots \ldots \ldots \ldots \ldots$ & 800 & 1200 & & \\
\hline
\end{tabular}

Expérience $\mathrm{n}^{\circ} 3$ (tableau III). 26 rats (13 infestés et 13 non infestés). Nous déterminons le poids moyen commun à tous les animaux, qu'ils soient infestés ou non. Nous fixons une dose de 100 milliunités pour $100 \mathrm{~g}$ de cette valeur moyenne, ce qui, en valeur relative, correspond à $90 \mathrm{mU} / 100 \mathrm{~g}$ chez les témoins et à $110 \mathrm{mU} / 100 \mathrm{~g}$ chez les animaux parasités.

La corticostéronémie des témoins passe de 12 à 77,8 $\mu \mathrm{g}$ et celle des parasités de 9,3 à $58,8 \mu \mathrm{g}$.

\section{Test à la Métopirone.}

Expérience $\mathrm{n}^{\circ} 1$ (tableau IV): infestation par 16000 larves (40 rats); témoins (30 rats). Le test à la Métopirone est accompli au $15^{\circ}$ jour de l'infestation et avec une dose de $100 \mathrm{mg}$ de produit par kilo de poids à dix heures puis à midi. L'examen des chromatogrammes sur couche mince montre que les animaux infestés ou non répondent avec la même intensité. Le taux de la corticostérone, au moment de l'épreuve, est de $4,5 \mu \mathrm{g}$ (rats parasités) et de $8 \mu \mathrm{g}$ (rats témoins) ; les taches correspondant à la désoxycorticostérone peuvent être évaluées aux environs de $16 \mu \mathrm{g} / 100 \mathrm{ml}$ alors que la corticostérone n'apparaît qu'à l'état de traces. 
TABLEAU IV. - Test à la Métopirone (Epreuve $\mathrm{n}^{\circ} 1$ )

\begin{tabular}{|c|c|c|c|c|c|}
\hline & \multirow{2}{*}{$\begin{array}{l}\text { Temps } \\
\text { (jours) }\end{array}$} & \multicolumn{2}{|c|}{$\begin{array}{l}\text { Evolution du Parasitisme } \\
\text { (œufs }+ \text { larves } / g \text { de selles) }\end{array}$} & \multicolumn{2}{|c|}{$\begin{array}{l}\text { Corticostéronémie } \\
(\mathrm{ug} / 100 \mathrm{ml})\end{array}$} \\
\hline & & Témoins & Métopirone & Non infestés & Infestés \\
\hline 6 & $\ldots \ldots+\cdots, \ldots$ & 126000 & 156000 & & \\
\hline 8 & $\ldots, \ldots+\cdots, \cdots$ & 150000 & 161000 & & \\
\hline 9 & $\ldots \ldots \ldots \ldots \ldots$ & & & 8,2 & 6,3 \\
\hline 10 & n..., $\ldots$ & 112000 & 131200 & & \\
\hline 13 & 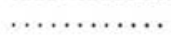 & 104000 & 80000 & 8 & 4,5 \\
\hline 15 * & n.w. & 29000 & 40400 & & \\
\hline 17 * & .......... & 11000 & 6400 & & \\
\hline 20 & $\ldots \ldots \ldots \ldots$ & 300 & 1200 & 9,5 & \\
\hline
\end{tabular}

Expérience $\mathrm{n}^{\circ} 2$ : infestation par 11000 larves (50 rats); témoins (30 rats). Pour pallier une insuffisance éventuelle d'action de la Métopirone, dans l'expérience précédente, l'expérimentation est étalée : 4 injections de $125 \mathrm{mg}$ de Métopirone $/ \mathrm{kg}$ toutes les trois heures réparties entre 8 heures et 17 heures. Le prélèvement sanguin est effectué à 19 heures, c'est-à-dire à une période où l'activité hypothalamo-pituitaire est encore importante ainsi que nous l'avons précédemment montré. Les résultats de cette épreuve confirment ceux précédemment obtenus dans des conditions différentes : la réaction des animaux infestés et celle des témoins sont du même ordre.

Le tableau $V$ synthétise l'ensemble des résultats correspondant aux tests d'exploration fonctionnelle.

TABLEAU V. - Exploration fonctionnelle de l'axe hypothalamo-hypophyso-cortico-surrénal (Corticostéronémie en $\mu \mathrm{g} / 100 \mathrm{ml}$ )

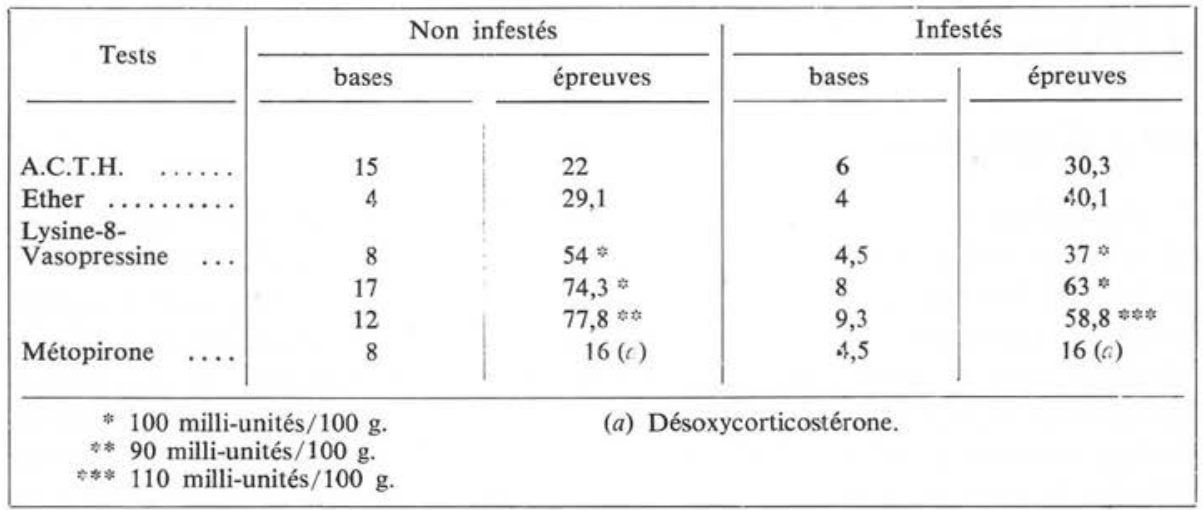




\section{Discussion}

L'épreuve à l'A.C.T.H. est en faveur d'une possibilité réactionnelle du cortex surrénal. Il semblerait donc que l'hypocorticostéronémie ne soit pas due à une incapacité fon:tionnelle de la corticosurrénale, mais soit provoquée par une excitation insuffisante de cette glande par sa stimuline.

La positivité du test à l'éther est en faveur de l'intégrité du couple hypothalamohypophysaire.

Pour Matsuda (1964), cet anesthésique agirait au niveau de l'éminence médiane. Cette région paraît avoir une très grande importance pour la neuro-sécrétion hypothalamique.

On peut donc considérer, dans l'état actuel de nos connaissances, que le stress à l'éther explore les possibilités sécrétrices de l'hypothalamus par excitation directe des centres diencéphaliques. C'est dire, en d'autres termes, que cette stimulation, qui physiologiquement devrait avoir lieu, ne semble pas se produire dans l'organisme hébergeant Strongyloïdes ratti. On peut donc penser à une inhibition de la neuro-sécrétion qui a été levée par l'inhalation d'éther.

Du test à la Lysine-Vasopressine se dégagent deux faits :

$1^{\circ}$ Dans les trois expériences, l'injection de Lysine-Vasopressine déclenche une hypercorticostéronémie. L'axe hypothalamo-hypophyso-surrénal présente donc les aptitudes fonctionnelles pour répondre à son excitation.

$2^{\circ}$ Dans les deux premières expériences, l'hypercorticostéronémie qui succède à l'injection de Lysine-Vasopressine est nettement plus importante chez les animaux témoins que chez ceux hébergeant le parasite.

On peut se demander si la dose injectée n'est pas responsable de cette différence. En effet, les animaux infestés pèsent sensiblement moins que les témoins. Or, la dose de L.V.P. étant fonction du poids, nous avons été amenés dans ces deux expériences à injecter, en valeur absolue, des quantités de produits inférieures aux rats malades.

La troisième expérience tient compte de cette remarque et la même dose de L.V.P. est inoculée à tous les animaux de sorte que, par suite des différences pondérales qui découlent du parasitisme, les animaux témoins reçoivent, en valeur relative, une quantité moindre que les rats infestés. Malgré cela, la réaction de ces derniers est toujours moins importante que celle des témoins.

Ces épreuves apportent la notion effective d'une inhibition d'origine parasitaire qui est telle qu'elle atténue l'action de la Lysine-Vasopressine.

Si l'on admet, avec la plupart des auteurs, que l'intervention de cette substance se situe au niveau de l'hypothalamus et provoque la sécrétion du cortico-releasingfactor, on obtient confirmation de la conclusion à laquelle nous a conduits le test à 
l'éther: c'est au niveau de la neuro-sécrétion que le parasitisme exerce son effet inhibiteur. En fait, selon Wied (1964), les points d'action de la Lysine-Vasopressine seraient multiples: centres nerveux supérieurs, hypothalamus et pituitaire. A ce niveau, l'influence porterait tout à la fois sur la synthèse et la libération d'A.C.T.H.

D'après le test à la Métopirone, la régulation par rétrocontrôle hormonal semble pouvoir s'exercer normalement, ce qui est en faveur de l'intégrité fonctionnelle de l'axe hypothalamo-pituitaire.

En fait, cette régulation est inhibée puisque la corticostéronémie des animaux porteurs de Strongles décroît progressivement. Les résultats du test à la Lysine-8Vasopressine permettent de la concevoir en révélant qu'un freinage semble s'exercer au niveau hypothalamique.

La Métopirone rend possible cette régulation car elle n'agirait pas seulement à la périphérie en supprimant la 11- $\beta$-hydroxylation, mais elle interviendrait aussi directement en déprimant les centres nerveux et plus particulièrement les noyaux hypothalamiques médians et l'hippocampe. Or, cette région de l'hippocampe est bien connue pour son action freinatrice sur la libération d'A.C.T.H. En levant l'inhibition notamment exercée par l'hippocampe, la Métopirone rend possible le rétrocontrôle hormonal.

Ainsi, l'hypocorticostéronémie provoquée par Strongyloides ratti serait la conséquence d'une diminution de la sécrétion hypothalamique, laquelle résulterait d'une inhibition du mécanisme nerveux qui la conditionne.

Entre les deux possibilités d'une dépression des voies stimulantes ou d'une excitation des voies freinatrices, le test à la Métopirone nous orienterait vers cette dernière.

Il reste à préciser le mécanisme par lequel le parasitisme interviendrait sur les centres nerveux responsables d'une inhibition du fonctionnement hypothalamo-pituitaire.

\section{REMERCIEMENTS}

La souche de Strongyloides ratti est entretenue à l'Unité de Recherche sur l'Immunologie des infections parasitaires (I.N.S.E.R.M.), 33000 Bordeaux. Nous remercions les Professeurs Pautrizel et Tribouley d'avoir bien voulu la mettre à notre disposition.

Nous remercions les Laboratoires Pharmaceutiques qui ont gracieusement mis à notre disposition les produits qui nous ont servi à réaliser cette expérimentation :

Laboratoire Endopancrine: Tétracosactide - zinc.

Laboratoire Sandoz: Lysine - Vasopressine.

Laboratoire Ciba: Métopirone.

Ce travail a pu être réalisé grâce à une subvention de la Fondation pour la Recherche médicale française. 


\section{Bibliographie}

Bailenger (J.) et Carcenac (F.) 1974. - Répercussions du parasitisme par Strongyloides ratti sur la sécrétion des gluco-cortico-stéroides chez le Rat. International $J$. Parasit., 4, 307-310.

Beigelman (P. M.), Slusher (M. A.), Slater (G. C.) et Roberts (S.), 1956. - Effect of anesthetics and collection time on corticosteroid secretion by Rat adrenal. Proc. Soc. exp. biol., 93, 608-611.

Cheifetz (P. N.), Gaffud (N. T.) et Ding Man (J.F.), 1969. - The effect of LysinVasopressin and hypothalamic extracts on the rate of corticosterone secretion in rats treated with Dexamethasone and Pentobarbitone. J. Endocr., 43, 521-528.

Clayton (G. W.), Libril (L.), Gardner (R. L.) et Guillemin (R.), 1963. - Studies on the circadian rhythm of pituitary adreno-corticotropic release in man. J. clin. Endocr., 23, 975-980.

Hedner (P.), 1961. - Expériences with a fluorometric method for determining corticosteroids in man rand Rat. Acta pharmacol., 18, 65-74.

HEDNER (P.), 1963. - Adrenocortical activity studied by determining plasma corticosteroids. Acta endocr. (suppl. 86).

Gwinup (G.), 1965. - Test for pituitary function using vasopressin. Lancet, 2, 572-573.

KiTAY (J. I.), 1961. - Sex differences in adrenal cortical secretion in the Rat. Endocrinology, 68, 818-824.

Matsuda (K.), Duyck (C.), Kendall (J.) et Greer (M. A.), 1964. — Pathways by which traumatic stress and ether induce increased A.C.T.H. release in the Rat. Endocrinology, 74, 981-985.

Van der Wal (B.), Wiegman (J.), Janssen (J.F.), Delver (A.) et de Wied (D.), 1965. Evaluation of pituitary adrenal function in children. Acta endocr., 48, 81-90.

DE WIED (D.), 1964. - The site of the blocking action of Dexamethasone on stressinduced pituitary A.C.T.H. release. J. Endocr., 29, 29-37. 